$@$ ERSpublications

PTTM is a rare, likely under-diagnosed cause of $\mathrm{PH}$, which is related to, but distinct from PTE http://ow.ly/OQad5

Neelam Kumar ${ }^{1}$, Laura C. Price ${ }^{2}$, M. Angeles Montero ${ }^{3}$, Konstantinos Dimopoulos ${ }^{2}$, Athol U. Wells ${ }^{1}$ and S. John Wort ${ }^{2}$ ${ }^{1}$ Interstitial Lung Disease Unit, Royal Brompton Hospital, London. ${ }^{2}$ Pulmonary Hypertension Centre, Royal Brompton Hospital, London. ${ }^{3}$ Dept of Histopathology, Royal Brompton Hospital, London.

Correspondence: Stephen John Wort, Pulmonary Hypertension Service, Royal Brompton Hospital, Sydney Street, London SW3 6NP, UK. E-mail: s.wort@imperial.ac.uk

Received: Jan 132015 | Accepted after revision: June 22015 | First published online: July 232015

Conflict of interest: Disclosures can be found alongside the online version of this article at erj.ersjournals.com

\title{
References
}

1 Simonneau G, Gatzoulis MA, Adatia I, et al. Updated clinical classification of pulmonary hypertension. J Am Coll Cardiol 2013; 62: Suppl. 25, D34-D41.

2 von Herbay A, Illes A, Waldherr R, et al. Pulmonary tumor thrombotic microangiopathy with pulmonary hypertension. Cancer 1990; 66: 587-592.

3 Dorfmuller P, Gunther S, Ghigna MR, et al. Microvascular disease in chronic thromboembolic pulmonary hypertension: a role for pulmonary veins and systemic vasculature. Eur Respir J 2014; 44: 1275-1288.

4 Uruga H, Fujii T, Kurosaki A, et al. Pulmonary tumor thrombotic microangiopathy: a clinical analysis of 30 autopsy cases. Intern Med 2013; 52: 1317-1323.

5 Abe H, Hino R, Fukayama M. Platelet-derived growth factor-A and vascular endothelial growth factor-C contribute to the development of pulmonary tumor thrombotic microangiopathy in gastric cancer. Virchows Archiv 2013; 462: 523-531.

6 Montani D, Bergot E, Gunther S, et al. Pulmonary arterial hypertension in patients treated by dasatinib. Circulation 2012; 125: 2128-2137.

7 Ranchoux B, Gunther S, Quarck R, et al. Chemotherapy-induced pulmonary hypertension: role of alkylating agents. Am J Pathol 2015; 185: 356-371.

8 Tashima Y, Abe K, Matsuo Y, et al. Pulmonary tumor thrombotic microangiopathy: FDG-PET/CT findings. Clin Nucl Med 2009; 34: 175-177.

9 Seki Y, Gotou M, Yasuda A, et al. [Secondary pulmonary hypertension due to pulmonary tumor thrombotic microangiopathy diagnosed by open lung biopsy.] Kyobu Geka 2011; 64: 916-919.

10 Uruga H, Morokawa N, Enomoto T, et al. [A case of pulmonary tumor thrombotic microangiopathy associated with lung adenocarcinoma diagnosed by CT-guided lung biopsy.] Nihon Kokyuki Gakkai Zasshi 2008; 46: 928-933.

11 Ota K, Matsuyama M, Kokuho N, et al. [An autopsy case of pulmonary tumor thrombotic microangiopathy complicated with interstitial pneumonia and lipoid pneumonia.] Nihon Kokyuki Gakkai Zasshi 2009; 47: 518-523.

12 Patrignani A, Purcaro A, Calcagnoli F, et al. Pulmonary tumor thrombotic microangiopathy: the challenge of the antemortem diagnosis. J Cardiovasc Med 2014; 15: 828-833.

13 Ogawa A, Yamadori I, Matsubara O, et al. Pulmonary tumor thrombotic microangiopathy with circulatory failure treated with imatinib. Intern Med 2013; 52: 1927-1930.

\section{Switching to nintedanib after discontinuation of pirfenidone due to adverse events in IPF}

\author{
To the Editor:
}

Recently two novel drugs, pirfenidone and nintedanib have been licenced for the treatment of idiopathic pulmonary fibrosis (IPF), marking a turning point in this previously untreatable, fatal disease.

Pirfenidone is a small molecule drug with anti-inflammatory and antifibrotic action. Its target molecule has not been identified, but preclinical studies have shown that it reduces transforming growth factor- $\beta$, platelet-derived growth factor (PDGF), fibroblast growth factor (FGF) as well as tumour necrosis factor $\alpha$, interleukin $1 \beta$ and monocyte chemotactic protein 1 [1]. Nintedanib is a multikinase inhibitor of vascular endothelial growth factor, PDGF and FGF [2], thus targeting several molecular pathways involved in the pathogenesis of IPF. These drugs are effective at reducing lung function decline in IPF [3-5]. However, 
both can have substantial adverse effects leading to discontinuation of treatment in approximately $15 \%$ of the patients with pirfenidone [3] and 19\% with nintedanib treatment [4] within studies. The adverse effect profiles show some overlap regarding gastrointestinal side-effects and elevation of liver enzymes. Furthermore, individual response to pirfenidone treatment is variable, with progression of fibrosis despite pirfenidone treatment in some patients, while most patients remain or become stable [6].

As in Europe, pirfenidone was first licensed in 2012. Most patients at our centre who were eligible for treatment were started on pirfenidone. Now that nintedanib, in 2015, has become available as a second treatment option the question of switching treatment arises in patients who either have marked persisting adverse effects or progressive disease despite treatment. The aim of this study was to assess the tolerability and efficacy of nintedanib treatment in IPF patients who discontinued previous pirfenidone treatment due to adverse events including rapid progression under treatment. In this preliminary report we provide data from seven patients, intending to point directions and ideas for further research needed in this area.

All patients from the Comprehensive Pneumology Center, (Munich, Germany) who were included in the nintedanib Compassionate Use Programme (CUP) (Boehringer Ingelheim study number: 1199.191) and previously treated with pirfenidone were identified and analysed retrospectively. Inclusion criteria for the nintedanib CUP programme were age $>40$ years, diagnosis of IPF in accordance with the guidelines [7], diffusing capacity of the lung for carbon monoxide (DLCO) $30-79 \%$ pred, and forced vital capacity $(\mathrm{FVC}) \geqslant 50 \%$ pred. The decision to offer nintedanib treatment was taken by the treating physician and was solely based on a clinical ground. Data analysis was undertaken under existent ethical votes. As part of their regular quarterly visits patients were assessed by: clinical examination, blood tests, lung function testing and blood gas analysis at three time-points. The three time-points were: time-point 1 under ongoing pirfenidone treatment 6 months prior to switching to nintedanib; time-point 2: 6 months later when inclusion into nintedanib CUP was effected; and time-point 3: after 6 months of nintedanib treatment (figure 1a). To assess occurrence and severity of adverse events under pirfenidone and nintedanib treatment respectively, a standardised questionnaire, based on the adverse events observed within clinical studies and Common Terminology Criteria for Adverse Events grading [8], was used. Rapid progression as an adverse event was defined as an absolute decline in FVC of $>10 \%$ within 6 months and, in this case, treatment was stopped due to clinical ineffectiveness. Beyond this, in the treatment-response analysis, clinical stability was defined as a relative semi-annual decline of FVC $<5 \%$ as previously reported $[6,9]$.

We identified seven patients who were switched from pirfenidone to nintedanib due to adverse events. All of these were male; five were former and two were never smokers. At baseline the median (range) characteristics of the patients were as follows: age 77.1 years ( $44.8-83.8$ years), body mass index $23.9 \mathrm{Kg} \cdot \mathrm{m}^{-2}$ $\left(21.8-28.1 \mathrm{Kg} \cdot \mathrm{m}^{-2}\right)$, time since diagnosis 2.9 years $(0.9-8$ years), FVC $69 \%$ pred $(61-92 \%$ pred), DLCO $40.4 \%$ pred (29-48\% pred), arterial oxygen tension standard $52.7 \mathrm{mmHg}(49.3-72.7 \mathrm{mmHg})$. The median duration of treatment was 6 months for both drugs.

In two patients treatment with pirfenidone was stopped due to rapid progression of IPF under treatment (table 1). Five patients did not tolerate the drug, despite trials of dosage reduction and supportive therapy. Interestingly, the most frequent adverse effect leading to discontinuation of pirfenidone treatment in four patients was general asthenia (table 1). Of note, no patient had elevated liver enzymes or rash given as the reason for the discontinuation of the drug.

Nintedanib treatment induced intolerable adverse effects that led to treatment discontinuation in one patient. This patient experienced similar adverse effects under both drugs with a combination of gastrointestinal disorders and severe asthenia; however, it was stated that asthenia was more pronounced under pirfenidone than nintedanib, while diarrhoea was the main reason for the discontinuation of nintedanib. As this patient completed no more than 1 month of both treatments, he was not in included in the response analysis. All the other four patients who had discontinued pirfenidone, due to intolerable adverse effects, displayed a better tolerability of nintedanib and continued this treatment. Gastrointestinal adverse effects were observed in all patients under nintedanib treatment, but were mild and manageable in six out of the seven patients.

Of the two patients who were switched due to rapidly progressive disease under pirfenidone, one experienced an acute exacerbation and died due to respiratory failure 3 months after the initiation of nintedanib treatment and the other patient had a further decline in lung function, although it was slower than before (figure 1a).

Regarding treatment response, two patients stabilised under nintedanib treatment, which had previously been unstable, one patient remained as stable as before after switching to nintedanib, two patients were unstable under both treatments, and one patient who had been stable under pirfenidone was unstable after an acute exacerbation under nintedanib (figure $1 b$ ). 
Pirfenidone and nintedanib have different pharmacodynamic and pharmacokinetic characteristics, but show some overlap in adverse events regarding gastrointestinal events and increases in liver enzymes. In the international CAPACITY (Clinical Studies Assessing Pirfenidone in Idiopathic Pulmonary Fibrosis: Research on Efficacy and Safety Outcomes) and ASCEND (Assessment of Pirfenidone to Confirm Efficacy and Safety in IPF) trials, the most frequent adverse event under pirfenidone was nausea and this was also the most frequent reason for treatment discontinuation $[3,5,10]$. Interestingly, in our patients asthenia and chronic fatigue was the most frequent reason for pirfenidone discontinuation, while this occurred with a frequency $<1.5 \times$ placebo in the pooled study analysis [10]. However, all of our patients noted a temporal link with the onset of treatment and an improvement of asthenia upon treatment discontinuation suggesting a causal relationship to the drug. Asthenia is a multifactorial syndrome that is frequently found in cancer [11, 12], but also in IPF and strongly affects quality of life [13]. Generally, the factors known to contribute to asthenia include cytokine secretion, anorexia, depression, hypothyroidism and anaemia [12]. Hypothyroidism and anaemia were excluded in our patients, but as anorexia was highly prevalent, this could be a contributing factor. Yet, in our patients anorexia was mild and only two out of six patients experienced weight loss. Another hypothetical mechanism might be an altered cytokine profile and the, so far unidentified, molecular target. Further studies should pay attention to frequency and mechanism of asthenia induced by pirfenidone.

In patients treated with nintedanib within clinical studies, the most common adverse event was diarrhoea, which was generally mild and manageable, and gastrointestinal disorders also accounted for the majority of treatment discontinuations [4]. These findings were also reflected in our patients.

The limitations of this study include its retrospective design and the small number of patients, all of whom were male and the majority at advanced age and disease stage. Moreover, the patients were selected for drug intolerability and we only looked at the switching from pirfenidone to nintedanib, but not vice versa. Hence, no conclusions can be drawn regarding the comparison of efficacy and tolerability between the two drugs in less selected and patients who did not undergo pretreatment. Recently, safety and pharmacokinetics of nintedanib when added to pirfenidone were evaluated [14]. In patients who display a good tolerability of one treatment, such combination therapy may be the next step in IPF treatment.

a)

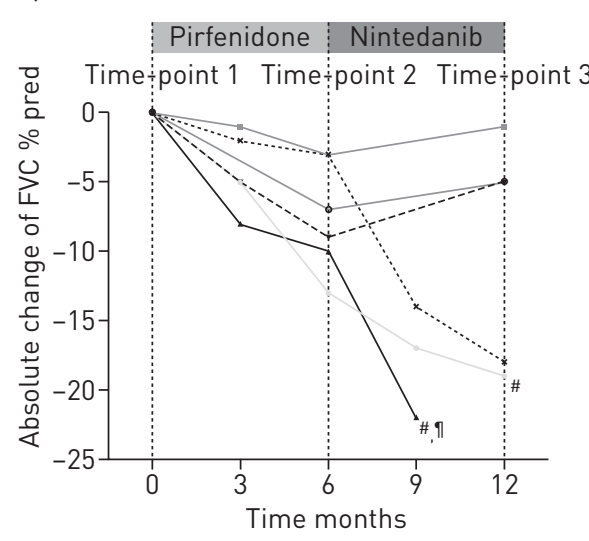

b)

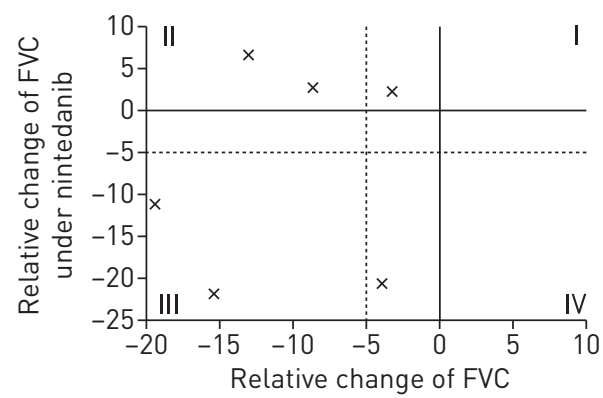

under pirfenidone

Quadrant Semi-annual decline of FVC with cut-off $>5 \%$

\begin{tabular}{lcl}
\hline Stable-stable & I & 1 \\
Unstable-stable & II & 2 \\
Unstable-unstable & III & 2 \\
Stable-unstable & IV & 1 \\
\hline
\end{tabular}

FIGURE 1 Intra-individual response to nintedanib in patients with interstitial pulmonary fibrosis (IPF) who discontinued pirfenidone treatment due to adverse events. a) Absolute change in forced vital capacity (FVC) under pirfenidone and nintedanib treatment. Each line represents one individual patient. Time-point 1, under ongoing pirfenidone treatment 6 months prior to switching to nintedanib; time-point 2, 6 months when inclusion into nintedanib Compassionate Use Programme (CUP) was effected; time-point 3, after 6 months of treatment with nintedanib. \#: these patients were switched to nintedanib due to rapid progression; ": this patient died due to respiratory failure in an acute exacerbation at 9 months. b) Relative change of FVC in response to 6 months of pirfenidone and nintedanib treatment. Stability is categorised according to semi-annual decline of FVC with a cut-off point of $5 \%$, represented by the dashed lines; crosses represent data from individual patients. The resulting subgroups are I: stable under pirfenidone and stable under nintedanib; II: unstable under pirfenidone and stable under nintedanib; III: unstable under pirfenidone and unstable under nintedanib; and IV: stable under pirfenidone and unstable under nintedanib. 


\section{TABLE 1}

Adverse events

\begin{tabular}{|c|c|c|c|c|c|c|c|c|c|c|c|}
\hline \multicolumn{6}{|c|}{ Pirfenidone } & \multicolumn{6}{|c|}{ Ninetedanib } \\
\hline \multirow[t]{2}{*}{ Subjects } & \multicolumn{5}{|c|}{ Grade } & \multirow[t]{2}{*}{ Subjects } & \multicolumn{5}{|c|}{ Grade } \\
\hline & 1 & 2 & 3 & 4 & 5 & & 1 & 2 & 3 & 4 & 5 \\
\hline
\end{tabular}

\begin{tabular}{|c|c|c|c|c|c|c|}
\hline \multicolumn{7}{|l|}{ Gastrointestinal } \\
\hline Diarrhoea & $1(14)$ & 1 & & $7(100)$ & 6 & 1 \\
\hline Nausea & $1(14)$ & & 1 & $2(29)$ & 1 & 1 \\
\hline Vomiting & $0(0)$ & & & $1(14)$ & 1 & \\
\hline Dyspepsia & $2(28)$ & 1 & 1 & $5(71)$ & 3 & 2 \\
\hline Anorexia & $6(86)$ & 4 & 11 & $3(43)$ & 1 & 2 \\
\hline Weight loss & 2 (29) & 1 & 1 & $2(29)$ & 2 & \\
\hline \multicolumn{7}{|l|}{ Skin } \\
\hline Pruritus & 2 (29) & & & $0(0)$ & & \\
\hline Rash & $1(14)$ & & & $0(0)$ & & \\
\hline Photosensitivity reaction & $3(43)$ & 3 & & $0(0)$ & & \\
\hline \multicolumn{7}{|l|}{ Other } \\
\hline Insomnia & $1(14)$ & & & $0(0)$ & & \\
\hline Dizziness & $3(43)$ & & & $2(29)$ & & \\
\hline Asthenia & $5(71)$ & & & $3(43)$ & & \\
\hline Arthralgia & $3(43)$ & 2 & 1 & $0(0)$ & & \\
\hline Pharyngolaryngeal pain & $0(0)$ & & & $1(14)$ & & \\
\hline Hot flush & $0(0)$ & & & $0(0)$ & & \\
\hline AST/ALT >3 times upper limit of normal & $0(0)$ & & & $0(0)$ & & \\
\hline Progression of IPF & $2(29)$ & & & $2(29)$ & & \\
\hline Acute exacerbation & $1(14)$ & & & $2(29)$ & & \\
\hline \multicolumn{7}{|l|}{ Serious AE } \\
\hline $\mathrm{AE}$ requiring hospitalisation & $2(29)$ & & & $2(29)$ & & \\
\hline Acute exacerbation & $2(29)$ & & & $2(29)$ & & \\
\hline$A E$ leading to treatment discontinuation & $7(100)$ & & & $2(29)$ & & \\
\hline Gastrointestinal disorders & $0(0)$ & & & $1(14)$ & & \\
\hline Asthenia/fatigue & $4(57)$ & & & 0 & & \\
\hline Arthralgia & $1(14)$ & & & 0 & & \\
\hline Exacerbation/progression & $2(29)$ & & & $1(14)$ & & \\
\hline
\end{tabular}

Data are presented as $\mathrm{n}(\%)$ or $\mathrm{n}$. Grade refers to the Common Terminology Criteria for Adverse Events grading [8]. AST: aspartate transaminase; ALT: alanine transaminase; IPF: idiopathic pulmonary fibrosis: AE: adverse events.

The present study indicates that in selected patients who do not tolerate pirfenidone, switching treatment to nintedanib may be well tolerated, despite similar adverse event profiles. Moreover, the intra-individual response to the two drugs may differ, as we identified patients who were stable under one drug treatment but were not under the other drug treatment. However, this study is of preliminary nature due to the small number of patients and its retrospective design. Further studies will be necessary to confirm our findings and evaluate whether individual treatment response may be predicted by molecular biomarkers, thereby favouring decision for one or the other drug as a first-line treatment.

0

@ERSpublications

Nintedanib can be well tolerated in patients who stopped pirfenidone treatment due to intolerable adverse effects http://ow.ly/OYEAK

Katrin Milger ${ }^{1}$, Nikolaus Kneidinger ${ }^{1}$, Claus Neurohr ${ }^{1}$, Frank Reichenberger ${ }^{2}$ and Jürgen Behr ${ }^{1,2}$

${ }^{1}$ Dept of Internal Medicine V, University of Munich, Comprehensive Pneumology Center, member of the German Center for Lung Research (DZL), Munich, Germany. ${ }^{2}$ Dept of Respiratory Medicine, Asklepios Clinic Gauting, Comprehensive Pneumology Center, member of the German Center for Lung Research (DZL), Munich, Germany.

Correspondence: Katrin Milger, Department of Internal Medicine V, Comprehensive Pneumology Center, Member of the German Center for Lung Research (DZL), University of Munich, Ziemssenstr. 1, 80336 Munich, Germany.

E-mail: Katrin.Milger@med.uni-muenchen.de

Received: April 132015 | Accepted after revision: June 072015 | First published online: Sept 042015

Conflict of interest: Disclosures can be found alongside the online version of this article at erj.ersjournals.com 


\section{References}

1 Schaefer CJ, Ruhrmund DW, Pan L, et al. Antifibrotic activities of pirfenidone in animal models. Eur Respir Rev 2011; 20: 85-97.

2 Hilberg F, Roth GJ, Krssak M, et al. BIBF 1120: triple angiokinase inhibitor with sustained receptor blockade and good antitumor efficacy. Cancer Res 2008; 68: 4774-4782.

3 Noble PW, Albera C, Bradford WZ, et al. Pirfenidone in patients with idiopathic pulmonary fibrosis (CAPACITY): two randomised trials. Lancet 2011; 377: 1760-1769.

4 Richeldi L, du Bois RM, Raghu G, et al. Efficacy and safety of nintedanib in idiopathic pulmonary fibrosis. $N$ Engl J Med 2014; 370: 2071-2082.

5 King TE Jr, Bradford WZ, Castro-Bernardini S, et al. A phase 3 trial of pirfenidone in patients with idiopathic pulmonary fibrosis. N Engl J Med 2014; 370: 2083-2092.

6 Loeh B, Drakopanagiotakis F, Bandelli GP, et al. Intraindividual response to treatment with pirfenidone in idiopathic pulmonary fibrosis. Am J Respir Crit Care Med 2015; 191: 110-113.

7 Raghu G, Collard HR, Egan JJ, et al. An official ATS/ERS/JRS/ALAT statement: idiopathic pulmonary fibrosis: evidence-based guidelines for diagnosis and management. Am J Respir Crit Care Med 2011; 183: 788-824.

8 US department of Health and Human Services. Common Terminology Criteria for Adverse Events (CTCAE) Version 4.0. Washington, US Department of Health and Human Services, 2009. Available from: http://evs.nci.nih. gov/ftp1/CTCAE/CTCAE_4.03_2010-06-14_QuickReference_5x7.pdf

9 Richeldi L, Ryerson CJ, Lee JS, et al. Relative versus absolute change in forced vital capacity in idiopathic pulmonary fibrosis. Thorax 2012; 67: 407-411.

10 Valeyre D, Albera C, Bradford WZ, et al. Comprehensive assessment of the long-term safety of pirfenidone in patients with idiopathic pulmonary fibrosis. Respirology 2014; 19: 740-747.

11 National Comprehensive Cancer Network. NCCN Guidelines: Cancer-Related Fatigue Version 02. NCCN, 2015. Available from: http://www.nccn.org/professionals/physician_gls/f_guidelines.asp

12 Mitchell SA, Berger AM. Cancer-related fatigue: the evidence base for assessment and management. Cancer $J$ 2006; 12: 374-387.

13 Swigris JJ, Kuschner WG, Jacobs SS, et al. Health-related quality of life in patients with idiopathic pulmonary fibrosis: a systematic review. Thorax 2005; 60: 588-594.

14 Ogura T, Taniguchi H, Azuma A, et al. Safety and pharmacokinetics of nintedanib and pirfenidone in idiopathic pulmonary fibrosis. Eur Respir J 2015; 45: 1-12. 